Research Article

\title{
Global Information Management Model and Identification Method Based on Deep Reinforcement Learning
}

\author{
RuiLe Lan $^{1}$ and XiJia He $\mathbb{i D}^{2}$ \\ ${ }^{1}$ Network Information Center, Nanning Normal University, Guangxi, Nanning 540001, China \\ ${ }^{2}$ School of Information and Management, Guangxi Medical University, Nanning 530021, Guangxi, China \\ Correspondence should be addressed to XiJia He; hexijia@gxmu.edu.cn
}

Received 22 September 2021; Revised 12 October 2021; Accepted 13 October 2021; Published 27 October 2021

Academic Editor: Gengxin Sun

Copyright (c) 2021 RuiLe Lan and XiJia He. This is an open access article distributed under the Creative Commons Attribution License, which permits unrestricted use, distribution, and reproduction in any medium, provided the original work is properly cited.

\begin{abstract}
Many countries in the world are actively conducting research on information technology and management. And deep learning is a new type of efficient and open technology system and tool. It develops and utilizes information and provides new knowledge based on the information processing capabilities of the human brain. Its essence lies in understanding the inner connection of things and thinking about problems by obtaining relevant data, so as to achieve the learning goal. Therefore, the research on global information management models and identification methods in this article is based on the consideration of global information security and is to create a good global environment. This article mainly uses the data analysis method, system construction method, and investigation method to sort out and study the content of this article. The survey results show that 270 people put the focus of global information on the ecological environment, and about $30 \%$ of them attach great importance to the security of global information.
\end{abstract}

\section{Introduction}

In recent years, the development of information technology has been very fast, and it has had a huge impact on human society. With the expansion of the scale of global information resources, it has a profound impact on human social life, economy, and other aspects. Among them, network security and communication technology are the biggest challenges and opportunities currently facing the world. Therefore, this article is based on deep reinforcement learning to explore global information management models and identification methods in order to secure and optimize global information management. Communication engineering studies the transmission of information in the form of electromagnetic wave, acoustic wave, or light wave from the transmitting end (source) to one or more receiving ends (sinks) through electrical pulse. Whether the receiver can correctly identify the information depends on the loss power in transmission. Signal processing is an important part of communication engineering, which includes filtering, coding, and decoding.
There are many studies on global information management models and identification methods. For example, Soldier Sun said that with the continuous progress and development of scientific information technology, it has promoted the rapid arrival of the era of big data. In order to effectively manage data, a big data management model needs to be established on its basis [1]. Tan Junjie believes that, in the era of information explosion, the proliferation of wireless communication terminals has led to a dramatic increase in the scale of networks. The emergence of artificial intelligence and machine learning has solved the problems of traditional manual modeling management methods [2]. Li Mengmeng said that information systems are extremely risky. Therefore, how to identify the security of the information system to ensure that the system can operate stably is a problem that requires serious consideration [3]. Therefore, this article is a new attempt to study deep reinforcement learning and global information management models and identification methods. Artificial intelligence is a branch of computer science. It attempts to understand the essence of intelligence 
and produce a new intelligent machine that can respond in a similar way to human intelligence. The research in this field includes robot, language recognition, image recognition, natural language processing, and expert system.

This article first studies machine learning and deep reinforcement learning and then studies a recognition method, namely, the voice recognition method. The following study described security information, which further explained the importance of security construction in global information construction. Finally, the relevant system is designed and module analyzed, and the questionnaire survey is carried out to obtain the results. Speech recognition technology is a high technology that enables the machine to convert the speech signal into the corresponding text or command through the process of recognition and understanding. Speech recognition technology mainly includes feature extraction technology, pattern matching criteria, and model training technology.

\section{Global Information Management Model and Identification Method Based on Deep Reinforcement Learning}

\subsection{Deep Reinforcement Learning}

2.1.1. Machine Learning and Reinforcement Learning. The core purpose of machine learning is to learn a certain concept from the data, or learn the rules in the data, perform pattern recognition on the data, and then use this concept to complete certain tasks. Different from the other two branches of machine learning, the idea of reinforcement learning comes from the use of trial and error and rewards to train the agent's learning behavior $[4,5]$. The basic environment of reinforcement learning is a Markov decision process. Reinforcement learning consists of four basic elements: strategy, reward, value function, and model. The most famous reinforcement learning algorithm is the Q-learning algorithm. The formula is as follows:

$$
r(a, b) \leftarrow r\left(a_{1}, b_{1}\right)+\beta\left[T_{s+1}+\lambda \max _{x} r\left(a_{s+1}, b\right)-r\left(a_{s}, b_{s}\right)\right] .
$$

If there is no $\max _{x} r\left(a_{s+1}, b\right)$ operation, Q-learning becomes another algorithm.

Note that reinforcement learning is a reward guiding behavior that agents learn in the way of "trial and error" and interact with the environment. The goal is to maximize the reward for agents. Reinforcement learning is different from supervised learning in connectionist learning, which is mainly reflected in strengthening signals. In reinforcement learning, the reinforcement signal provided by the environment is an evaluation of the quality of the action.

2.1.2. Deep Learning. The essence of deep learning is a multilayer neural network, and the function of the neural network is to estimate the unknown function. When the level is deepened, the network's estimated value of the function will be more accurate. As far as the current deep learning theory is concerned, deep learning network structures are mainly divided into three types: feedforward neural network, recurrent neural network, and convolutional neural network. The structure of convolutional neural network mainly includes convolutional layer, pooling layer, excitation unit, and fully connected layer. The convolutional layer is the process of convolution between the image and the convolution kernel $[6,7]$. When inputting a two-dimensional image $\mathrm{P}$, the result of it and the convolution kernel $L$ is

$$
W(a, b)=(P \times L)(a, b)=\sum_{x} \sum_{y} P(a+x, b+y) L(x, y) .
$$

Deep learning emphasizes the depth of the model structure, which usually has 5, 6, or even 10 hidden layer nodes; the importance of feature learning is clarified. In other words, the feature representation of the sample in the original space is transformed into a new feature space through layer-by-layer feature transformation, so that it is easier to classify or predict; through the learning and tuning of the network, the functional relationship from input to output is established.

2.1.3. Deep Reinforcement Learning. The main motivation for the transition from reinforcement learning to deep reinforcement learning is that in a complex environment, the $\mathrm{t}$-value storage space will be large, which affects the computational cost and learning efficiency. A neural network to approximate the value of $t$ is used, that is,

$$
t(c, d, e) \approx t^{\mu}(c, d)
$$

The essence of deep reinforcement learning is still reinforcement learning, but a deep neural network is added to extract features. From the traditional reinforcement learning span to the deep reinforcement learning process, the main problem is stability $[8,9]$.

The learning process of deep reinforcement learning can be described as follows: the agent interacts with the environment to obtain a high-dimensional observation at each time and uses the deep learning method to perceive the observation to obtain the specific state feature representation; the value function of each action is evaluated based on the expected return, and the current state is mapped to the corresponding action through some strategy; and the environment reacts to this action and gets the next observation. By continuously cycling the above process, we can finally get the optimal strategy to achieve the goal.

\subsubsection{Commonly Used Deep Learning Models.}

(1) Auto encoder model: it is a neural network that tries to make its output equal to the input. It is an unsupervised learning algorithm that does not need to use classification labels for nonlinear feature extraction.

(2) Convolutional neural network: it is composed of multiple adjacent convolutional layers and down- 
sampling layers. The layers of the network are not linearly mapped. The characteristics of the image are from the convolutional layer to the down-sampling layer. This process is down-sampling, and the the sampling layer reaches the convolutional layer.

(3) Recurrent neural network: recurrent neural network is another common deep network structure. Its characteristic is that the network will calculate the current output information based on the information at the previous moment in the sequence $[10,11]$. The core of recurrent neural network is composed of hierarchical nodes, in which the high-level nodes are the parent nodes, the low-level nodes are called child nodes, and the last child nodes are usually output nodes. The properties of nodes are the same as those in the tree.

2.2. Basics of Speech Recognition. In order to collect global security information at all times, this article uses voices that can appear at any time to recognize and generate data.

2.2.1. Voice Recognition Technology. Speech recognition technology mainly includes continuous speech recognition technology and keyword recognition technology. The continuous speech recognition system is mainly composed of four parts: signal processing and feature extraction, acoustic model, language model, and decoding search part, as shown in Figure 1.

According to Figure 1, we can know that the speech recognition process is as follows: first, input the signal, perform preprocessing, then perform feature extraction, and combine the acoustic models. During recognition, the extracted features are scored to generate acoustic model scores [12].

2.2.2. Speech Signal Feature Extraction. (1) Voice Signal Preprocessing. For the speech signal, after the short-time Fourier transform is performed, the high-frequency component is relatively small. Usually, a first-order high-pass digital filter is used to achieve the following equation:

$$
F(x)=1-\ell \mathcal{K}^{-1} .
$$

Among them, $\ell$ represents the pre-emphasis coefficient and the value range is between 0.9 and 1 .

Suppose the window function is $g$ and the voice signal is $h$, then the voice signal after windowing is as follows:

$$
H_{g}(m)=g(m) \bullet h(m) .
$$

(2) Common Feature Extraction Methods. The steps to extract features are as follows:

(i) The input speech signal is preprocessed to obtain the time-domain signal that is framed and windowed.

(ii) A short-term Fourier transform is performed on each speech signal in the windowed time domain to obtain its linear frequency spectrum. (iii) Front-end processing refers to processing the original speech before feature extraction to partially eliminate the influence of noise and different speakers, so that the processed signal can better reflect the essential characteristics of speech. The most commonly used front-end processing is endpoint detection and speech enhancement. Endpoint detection is to distinguish the speech and nonspeech signal periods in the speech signal, and accurately determine the starting point of the speech signal.

2.2.3. Acoustic Model and Language Model. (1) Acoustic Model Modeling Unit. The first is to select the appropriate modeling unit and then select the modeling unit. After triphone modeling, the state needs to be bound. Hidden Markov model can be used for speech recognition. The probability parameter can be expressed as shown in Figure 2.

(2) Language Model. Given a historical word sequence $k$, the probability $\mathrm{P}(x \mid k)$ of the appearance of the current word $x$ is calculated and the number of occurrences of the historical word $b_{1}$ sequence and the number of occurrences of the historical word $b_{2}$ sequence and the current word are counted; then,

$$
P(x \mid k)=\frac{b_{1}}{b_{2}}
$$

2.2.4. Decoder. The dynamic decoding network connects the same words in the pronunciation dictionary in parallel. Commonly used dictionaries include linear dictionaries and tree dictionaries, reducing the size of the state network search space and the amount of calculation. The static decoding network combines several models to form a state network. The input is a sequence of states, and the output is a sequence of words and scores.

\subsection{Modulation Signal Recognition Based on Convolutional Neural Network}

2.3.1. Multiscale Network Architecture. Convolutional neural networks use convolution kernels that are much smaller than the input image to reduce storage costs and increase computational efficiency, and the same parameters are used for a set of neural connections to reduce the number of parameters.

The multiscale convolution subnetwork, according to different regional features, respectively, design small-granularity multiscale convolution kernels to extract more detailed time-frequency map features. In the constructed network, the filter can realize cross-channel interaction and information fusion and, at the same time, can reduce the dimensionality of the convolution filter channel. When constructing DMCNN, the size and step size of the kernel are selected to ensure that each channel outputs the same size feature map, and finally all the features are merged at the parallel node to obtain the final feature map. The overall process can be expressed as shown in Figure 3. 


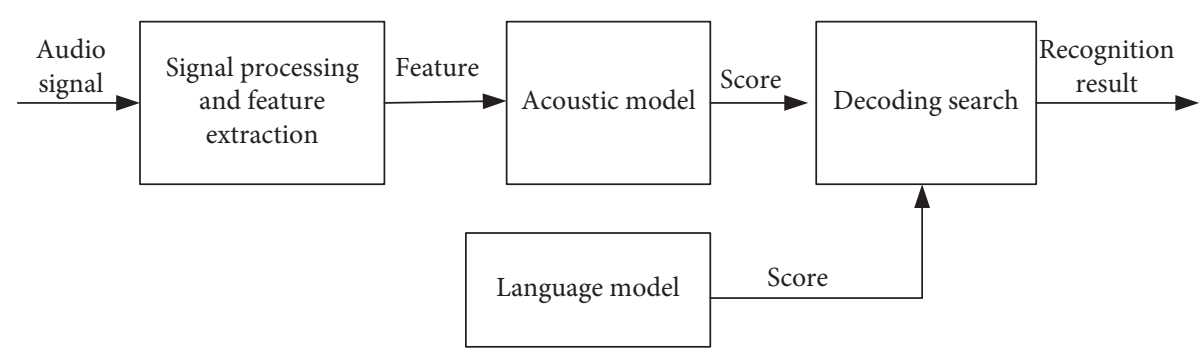

Figure 1: Speech recognition system.

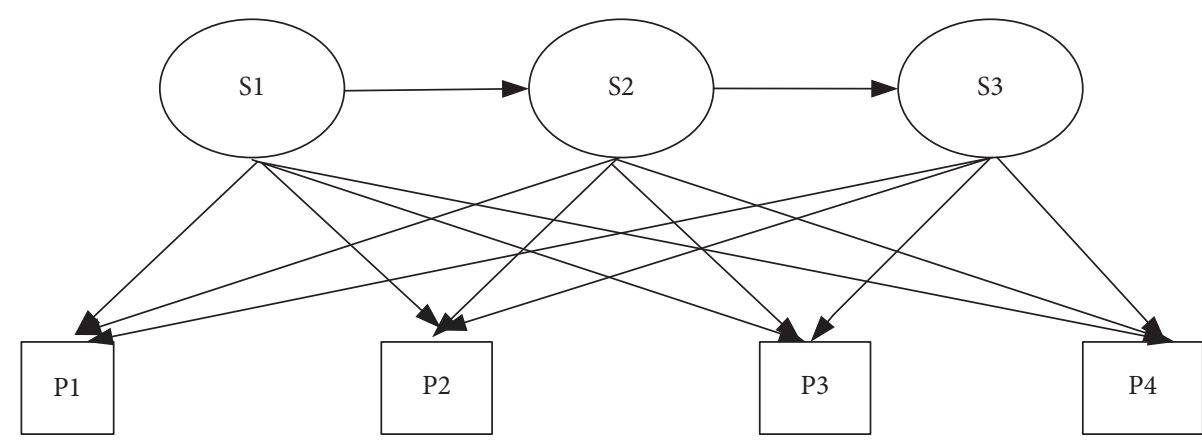

FIGURE 2: Hidden Markov probability parameters.

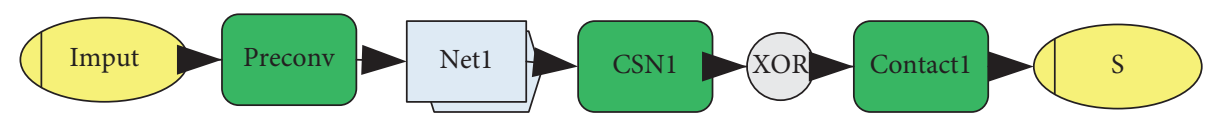

FIgURE 3: DMCNN algorithm flow.

The full connection layer in the convolutional neural network is equivalent to the hidden layer in the traditional feedforward neural network. The full connection layer is located at the last part of the hidden layer of convolutional neural network and only transmits signals to other full connection layers. The characteristic graph will lose its spatial topology in the full connection layer and be expanded into a vector and passed through the excitation function. According to the viewpoint of representational learning, the convolution layer and pooling layer in convolution neural network can extract the features of input data, and the function of full connection layer is to nonlinearly combine the extracted features to obtain the output; that is, the full connection layer itself is not expected to have the ability of feature extraction but tries to use the existing high-order features to complete the learning goal.

2.4. Information Security. Once network information is distributed, it is convenient to send and can no longer control the spread and use of information, and there is no void or recall mechanism. Moreover, in the Internet age, information is disseminated and stored in electronic form. Compared with any period in history, privacy protection and correct use of information are more important.

(1) Information security involves cryptography and designing and analyzing cryptographic schemes, and security protocol is one of the core research tasks of information security.

(2) In the world of computer networks, digital signatures are one of the effective means to ensure the integrity and nonrepudiation of electronic documents.

(3) The algorithms to realize digital signature mainly include RSA, DSS/DSA, and elliptic curve cryptography. Under the same security level, the ECC key length is smaller than the public key encryption system based on the finite field; the calculation amount is smaller, and the calculation speed is faster.

(4) The general term for the security infrastructure implements and provides general security services based on public key theory and technology and does not specifically refer to any specific cryptographic device and its security equipment. Through the security services provided by PKI, users can establish secure communications and network applications to transparently manage the passwords and certificates required for encryption, digital signatures, and other cryptographic services.

(5) The public key is related to the user's identity and can identify the user self-evidently; no public key directory is required; no online third-party service is required; only the trusted $\mathrm{PKG}$ generates the private key for the user when the user first joins. Therefore, 
IBC is far superior to PKI in key management and other aspects.

(6) Content extraction signature scheme: under the premise of following the extraction strategy, the signature holder blinds the subdocuments and extracts the unblinded subdocuments and their signatures.

(7) Encryption is one of the most commonly used solutions to protect information from unauthorized access, which is simple and effective. If the user attempts to access part of the encrypted information stored in the remote, the retrieved corresponding cipher text can be sent back to the local, processed, and operated after decryption in a trusted computing environment, and encrypted again and sent back to update the remote storage information. $\mathrm{PH}$ is a special encryption technology that allows direct operations on encrypted data without the need for decryption keys to participate.

One of the purposes of digital signature is to replace the traditional manual signature and seal in the network environment. It plays important roles: anti-impersonation: the private key is only known by the signer, so it is impossible for others to construct the correct private key. Identifiable: as the traditional manual signature is generally met directly by both parties, the identity can be clear. In the network environment, the receiver must be able to identify the identity claimed by the sender. Tamper-proof: antidamage to the integrity of information. Anti-repudiation: the digital signature can identify the identity, and it is impossible to pretend to be forged. Then, as long as the signed message is protected, it is like keeping the manually signed contract text; that is, retaining the evidence, the signer cannot repudiate. Confidentiality: with confidentiality assurance, interception attacks will fail. Ensure: the integrity of information transmission, identity authentication of the sender, and prevent denial in the transaction.

2.5. Analysis and Design of Global Information System Management Platform. In-depth examination of the most important links and management elements in information system management and system security work, technical tools and management tools must be applied to key activities such as the construction of computer systems and management systems.

2.5.1. Construction of Computer Systems and Management Systems. The construction can be carried out around the following four aspects:

(1) Information system management: the informatization and proceduralization of the information system management is realized, and the entire life cycle of the information system is integrated from project establishment, construction, operation, and maintenance to termination into effective management.

(2) Information security management: unified registration and management of various information security incidents are carried out, and information security emergency plans are managed by category.

(3) Self-evaluation of level protection: regarding hierarchical protection as the authoritative "ruler" of information system security, through self-assessment and evaluation of hierarchical protection of information systems, it provides a powerful tool for the normalized management of system security.

(4) Statistical analysis query: global leaders at all levels use graphical interfaces to timely and accurately grasp the overall situation of informatization construction between countries and the overall situation of information security.

2.5.2. System Architecture Design. The design of the information system management platform architecture integrates the consideration of business layering and technology layering and consists of four parts: business management layer, platform application support, information resources, and platform foundation.

The business management layer implements business logic collection and processing. The application support layer provides data exchange, data sharing, user management and authorization, and other auxiliary functions between platforms. The information resource layer is dominated by information resource locations, mainly storing information systems, grade protection standards, plan management, security incidents, etc. The basic layer of the platform maintains the normal operation of the basic hardware and network equipment of the platform.

2.5.3. Process Outline Design. The security management platform runs through the entire life cycle of the information platform. It uses a workflow engine to focus on the construction and operation, and maintenance of the information platform to ensure the standard compliance of the information platform and promote the standardization and standardization of platform security work. The main processes of this system include project management, security incidents, system management, emergency response plans, self-assessment and evaluation, and platform maintenance.

\subsection{Global Information Management Module Design}

2.6.1. Design Goals. The interface design is simple and practical

A variety of query methods can be provided

Users can modify their own information at any time

The announcement information is easy to view and share

Supports data inspection on user input data to ensure data integrity and security

The system is robust

2.6.2. Database Design. The basic design of the database can be seen in Table 1, which is the basic setting of some databases. 
TABLE 1: Basic database design.

\begin{tabular}{lccc}
\hline Data Item & Designation & Type & \\
& ID & Integer & Length \\
& Name & Character & 9 \\
& Sex & Character & 18 \\
Resume & Remark & Text & 2 \\
Data dictionary & User type & - & 22 \\
\hline
\end{tabular}

\subsubsection{Module Function Design and Realization}

(1) Design and Implementation of Login Module. This page includes five parts: administrator, browser, request processing database, database engine, and back-end database. The specific steps are open the browser, display the page, then $\log$ in, pass the verification information, request the database list, and obtain the information. After that, provide the user list, verify the information, and send it. Display the administrator page, manage information, save data, and return information.

(2) Design and Implementation of System Setting Module. Tool management department information settings include the name of the management department, information about the person in charge, contact number, contact address, e-mail address, website address, establishment time, and other information. In addition, there are settings for users, parameters, and software location information.

(3) Design and Implementation of User Management Module. The user management module is divided into two parts: user type management and user economic, political, cultural, ecological, and other aspects of management. In the user-type management, we can add user types and we need to enter the user type name and the number of borrowable tools. In the economic, political, cultural, and ecological management pages, tool information can be added, modified, or deleted. To add tool information, you need to fill in the tool bar code, tool name, tool type, tool department, tool location, and other information.

\section{Questionnaire Survey}

3.1. Questionnaire Design. In view of the research on the management module and identification method of global information, the contents of this questionnaire design include the following:

(1) Basic information of the person filling in the questionnaire.

(2) What are the aspects of global information?

(3) What are the most important design aspects of global information?

(4) What are the identification methods for daily contact?

(5) What needs to be paid attention to in the identification of information?
3.2. Questionnaire Process. In this questionnaire survey, 500 citizens of this city were randomly selected, mainly students and teachers at school, to fill in the questionnaire on their information on interstate information. A total of 500 questionnaires were distributed, and people were invited to fill out the questionnaire online. The questionnaire process lasted for one month, effectively collecting 400 pieces of data, and the recovery rate reached $80 \%$. Data statistics and analysis are carried out in Section 4 of this article.

3.3. Questionnaire Statistics. Due to the relatively large amount of data in this questionnaire, computer system calculation methods are used to perform statistics and analysis on the data.

\section{Analysis of the Results of the Questionnaire}

4.1. Respondents' Support for Global Information. According to the results of the survey, it is generally believed that global information includes economic, political, cultural, and ecological aspects. However, different people have different views on these four aspects of global information management attitudes. The details are shown in Table 2:

From Figure 4, we can judge that ecological global information management is generally supported by different people. Ecology is an important foundation related to global living conditions, so it is essential for the ecological exchange of global information. Global management in economics, politics, and culture has reasonable parts but also uncertain factors. Because of the contradictions between countries, there will be conflicts in economics, politics, and culture.

4.2. Understanding of Information Recognition Methods. According to the survey results, there are many recognition methods, among which are voice recognition, image recognition, radio frequency recognition, and fingerprint recognition. These methods are usually used in smart devices. Applying it to global information management, people generally believe that security, information flow, and shareability are more important. The details are shown in Table 3.

As shown in Figure 5, we can see that security in global information exchange is the most important thing people value. This means that, in global information management, the protection of security performance is in the first place. In addition, the sharing of information is a major consideration in the establishment of a global 
TABLE 2: Respondents' supportive attitudes towards global information.

\begin{tabular}{lcccc}
\hline & Economic & Politic & Cultural & Ecology \\
\hline Male & 80 & 30 & 50 & 100 \\
Female & 40 & 20 & 30 & 50 \\
Students & 50 & 40 & 60 & 70 \\
Teachers & 40 & 35 & 55 & 50 \\
\hline
\end{tabular}

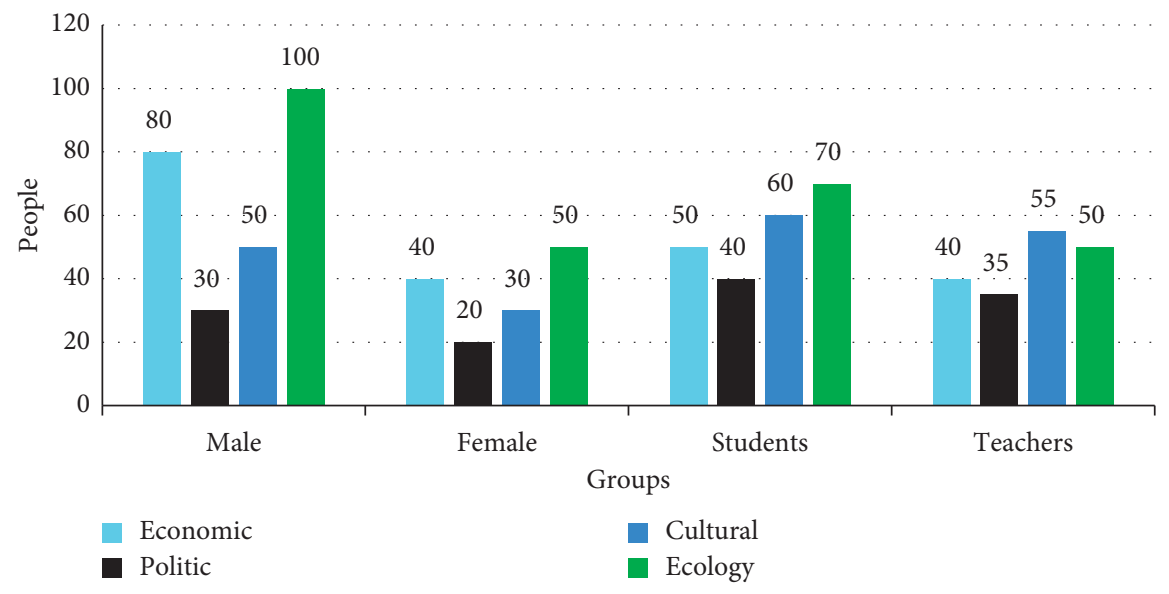

FIGURE 4: Respondents' supportive attitudes towards global information.

TABLE 3: Key issues of information recognition method.

\begin{tabular}{lccc}
\hline & Security (\%) & Mobility (\%) & Shareability (\%) \\
\hline Speech recognition & 30 & 15 & 20 \\
Image recognition & 35 & 25 & 19 \\
Radio frequency identification & 28 & 16 & 17 \\
Fingerprint recognition & 27 & 12 & 18 \\
\hline
\end{tabular}

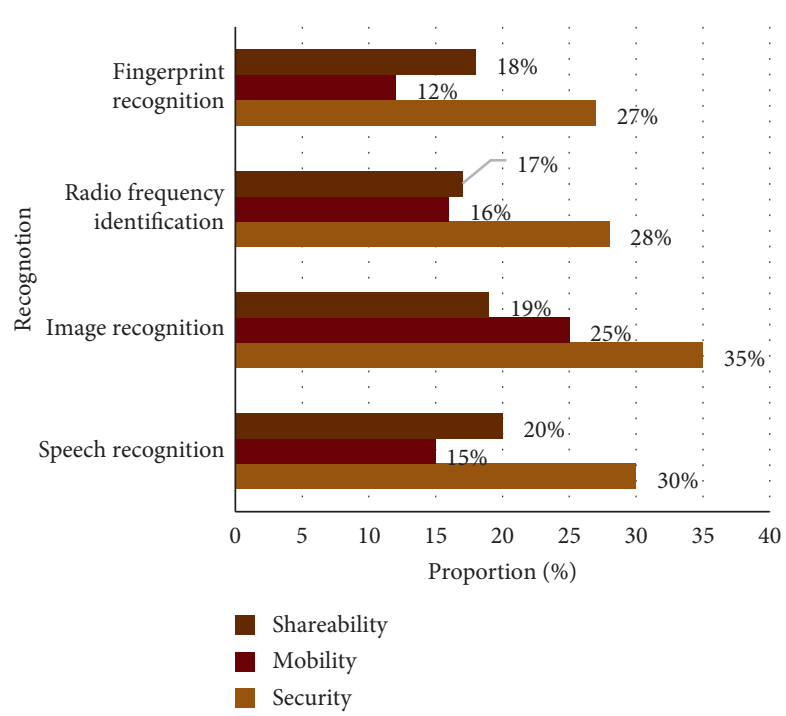

FIGURE 5: Key issues of information recognition method. 
information management system. Because global harmony requires all countries to work together.

\section{Conclusion}

Deep learning has been a hot research topic in recent years, and it has received extensive attention in the field of global information management. Deep learning is a new digital teaching model based on advanced theories such as network and multimedia technology. Human society is gradually entering the "big data era" and is accelerating the transition and innovation stage to the new economic model. Therefore, based on the design of the global information management system, it is necessary to apply deep reinforcement learning technology to system research to promote the unity and sharing, safety, and harmony of the global information system.

\section{Data Availability}

The experimental data used to support the findings of this study are available from the corresponding author upon request.

\section{Conflicts of Interest}

The authors declare that they have no conflicts of interest to report regarding the present study.

\section{Acknowledgments}

This work was supported by the Guangxi Key Research and Development Program, Topic: "Internet +" The construction and application of innovative design method system in educational service industry, Subject ID: AB18126068; Guangxi Education Science "12th Five-Year Plan” Education Planning Program, Topic: Research on the construction and application of public service platform for education management in Guangxi, Subject ID: 2015ZKY12; Academic Degree and Postgraduate Education Reform Project of Guangxi, Topic: The Exploration and Practice of the Collaborative Innovation Mechanism of Public Management Discipline under the Integration of industry-education Background, Subject ID: JGY2020044.

\section{References}

[1] S. Sun, "Research on big data management model based on data mining," Information Recording Materials, vol. 20, no. 2, pp. 60-62, 2019.

[2] J. Tan and Y. Liang, "Deep reinforcement learning method for intelligent communication," Journal of University of Electronic Science and Technology of China, vol. 49, no. 2, pp. 169-181, 2020.

[3] M. Li, "Talking about the method of information system security identification[J]," Science \& Technology Economic Guide, vol. 3, p. 28, 2018.

[4] C. Yu, S. Hu, and P. Ye, "Research on query expansion model based on deep reinforcement learning," Information Theory and Practice, vol. 42, pp. 150-157, 2019.
[5] C. Li, L. Cao, and X. Chen, "Research on exploration strategy of deep reinforcement learning based on cloud reasoning model," Journal of Electronics and Information Technology, vol. 040, no. 1, pp. 244-248, 2018.

[6] X. Zhao and S. Wu, "A brief introduction to bird flock detection and application based on deep reinforcement learning," Science and Information Technology, vol. 29, pp. 22-23, 2018.

[7] Y. Qi and S. Huang, "Portfolio management based on deep reinforcement learning DDPG algorithm," Computer and Modernization, vol. 273, no. 5, pp. 97-103, 2018.

[8] F. Li, Y. Guan, and S. Wang, "Research on the data asset management model and value evaluation method of power supply enterprises from the perspective of information ecology," Information Science, vol. 37, no. 10, pp. 48-54, 2019.

[9] L. Xiao, T. Meng, and W. Wang, "Research on the identification of intelligence analysis methods based on deep learning-taking the field of security intelligence as an example," Data Analysis and Knowledge Discovery, vol. 3, pp. 24-32, 2019.

[10] Y. Wang, H. Luo, and Z. Li, "Research on the cross-border flow network of global science and technology leaders-_analysis of complex network big data based on bibliographic information metrology," Technology and Innovation Management, vol. 39, no. 5, pp. 521-529, 2018.

[11] Y. Wang, D. Tian, and L. Zhang, "Identification and realization of new capabilities of enterprises in an information environment," Science and Technology Management Research, vol. 38, no. 16, pp. 190-196, 2018.

[12] M. Zhang, "Research on asset information management model and application," China Construction Information Technology, vol. 24, pp. 71-73, 2018. 